\title{
POLARIZATION CHARACTERISTICS OF RADIATION OF ATOMIC ENSEMBLE UNDER COHERENT EXCITATION IN THE PRESENCE OF A STRONG MAGNETIC FIELD
}

\author{
A. G. Petrashen, N. V. Sytenko \\ ITMO University, 197101, St. Petersburg, Russia \\ apetrashen@mail.ru
}

PACS 30.80k, 32.60+i

DOI 10.17586/2220-8054-2015-6-1-122-132

Numerical solutions of the Liouville equation are used to study of the polarization characteristics of an ensemble of He atoms in $3^{3} S_{1}$ state in the presence of the strong magnetic field when the coherent population of this level is realized from $2^{3} P$ state.

Keywords: Coherens excitation, evolution of density matrix, interference effects.

Received: 20 October 2014 Revised: 27 October 2014

\section{Introduction}

Coherent excitation of levels belonging to a particular state, can lead to a number of interesting phenomena, for example - coherent population trapping (CPT) and electromagneticallyinduced transparency, which have been extensively studied for decades. Continuing interest in these phenomena is related not so much to the phenomena themselves, but rather their applications - lasing without inversion [1], laser cooling [2] and stored light [3]. If the system under consideration is not in the CPT state, the polarization characteristics of its radiation can be used for the diagnostics of the anisotropy of the processes occurring in that system. Previously, these characteristics have been used for diagnostics of ions drift velocities in gas discharge plasma [4], the proton and electron beams of the sun's chromosphere and the earth's atmosphere [5], [6], the magnetic fields of Z-pinch [7] and tokamak [8] hot plasma and cold plasma of the upper layers of Earth's atmosphere [9].

In general, for the investigation of CPT phenomena, the lower superfine levels of alkali atoms are used. According to the our viewpoint the polarization characteristics of other atoms for instance helium are of interest. The emission of helium can be observed in the solar atmosphere and if the observation take place nearby the sun spots strong magnetic field should be imposed on the system of observation [10].

Due to the fact that the considered phenomena are associated with coherent processes, a satisfactory description of these processes is only possible using the apparatus of the density matrix, allowing one to take into account correlation in the initial conditions.

Polarization characteristics of the system's radiation are related to its internal ordering, which can be changed by an external magnetic field. Studies of the effect of the magnetic field on the system can be done in two ways, for the first one, the field is turned on during registration of systems radiation [11], [12], in the second, as it will be assumed in this paper, the system is constantly under the influence of the field. In the latter case, the role of the constant magnetic field is reduced to the splitting of the fine or hyperfine structures levels, and 
to changing the symmetry of the problem in question. The result of these two factors will be discussed below.

\section{Description of polarization phenomena}

To describe the polarization phenomena, it's convenient to expand the system density operator over the irreducible tensor operators:

$$
\rho=\sum_{k, q} \rho_{q}^{k} T_{q}^{k \dagger}
$$

The coefficients of this expansion are known as a polarization moments and can be defined by the following formula:

$$
\rho_{q}^{k}(j)=\sum_{m, m^{\prime}}(-1)^{j-m^{\prime}}\left[\begin{array}{ccc}
j & j & k \\
m & -m^{\prime} & q
\end{array}\right](\rho)_{m, m^{\prime}},
$$

where $j$ denotes the magnitude of the angular momentum of the system under consideration, $m$ is the magnetic quantum number and the expression in square brackets is known as the Clebsch-Gordan coefficients [13].

The physical meaning of the polarization moments is that they determine the ordering of the angular momenta of the ensemble of particles, i.e. the ensemble average of the linear combinations of the components of the tensor, which is comprised of the components of the angular momentum. For example, such averages of first and second rank tensors can be implied [10]:

$$
\begin{gathered}
\left\langle j_{z}\right\rangle=\frac{\sqrt{2 j(2 j+1)(2 j+2)}}{2 \sqrt{3}} \rho_{0}^{1}, \\
\left\langle j_{ \pm}\right\rangle= \pm \frac{\sqrt{2 j(2 j+1)(2 j+2)}}{\sqrt{6}} \rho_{\mp}^{1}, \\
\left\langle 3 j_{z}^{2}-j^{2}\right\rangle=\frac{\sqrt{(2 j-1) 2 j(2 j+1)(2 j+2)(2 j+3)}}{2 \sqrt{5}} \rho_{0}^{2}, \\
\left\langle j_{z} j_{ \pm}+j_{ \pm} j\right\rangle=\mp \frac{\sqrt{(2 j-1) 2 j(2 j+1)(2 j+2)(2 j+3)}}{2 \sqrt{15}} \rho_{\mp 1}^{2}, \\
\left\langle j_{ \pm}^{2}\right\rangle=\frac{\sqrt{(2 j-1) 2 j(2 j+1)(2 j+2)(2 j+3)}}{\sqrt{30}} \rho_{ \pm 2}^{2} .
\end{gathered}
$$

Conversely, the polarization moments define intensity of the radiation polarized along the unit vector $\vec{e}_{\lambda}$ emitted by the transition from the excited level with angular momentum $j$ to the lower-lying state with angular momentum $j_{0}$

$$
I_{\lambda}=I_{0} S p\left(d_{\lambda} \rho d_{\lambda}^{*}\right)=I_{0} \sum_{k, q}(-1)^{k}\left|\left\langle j_{0}\|1\| j\right\rangle\right|^{2}\left\{\begin{array}{ccc}
1 & 1 & k \\
j & j & j_{0}
\end{array}\right\} \Phi_{k, q}\left(\vec{e}_{\lambda}\right) \rho_{-q}^{k}(j),
$$

where $I_{0}$ is a factor that depends on fundamental constants and frequency, $d_{\lambda}$ is the projection of the atomic dipole moment on the direction of the oath $\vec{e}_{\lambda},\left\langle j_{0}\|1\| j\right\rangle$ - reduced matrix element, an expression in the figure braces $6 j$-symbol [13], and $\Phi_{k, q}\left(\vec{e}_{\lambda}\right)$ denotes the socalled polarization tensor, which is determined by observation conditions and depends on $s_{q}^{(\lambda)}-$ 
expansion coefficients of the unit vector of the polarization direction of detected radiation by circular unit vectors:

$$
\Phi_{k, q}\left(\vec{e}_{\lambda}\right)=\sum_{q_{1}, q_{2}}(-1)^{1-q_{1}} s_{q_{1}}^{(\lambda) *} s_{q_{2}}^{(\lambda)}\left[\begin{array}{ccc}
1 & 1 & k \\
-q_{2} & q_{1} & q
\end{array}\right] .
$$

In particular, if $I_{x}, I_{y}, I_{z}$ would denote the intensities of the radiation polarized in three mutually perpendicular directions defined by the axes $O X, O Y$ and $O Z$ respectively, from formula (4) can be obtained:

$$
I_{x}+I_{y}+I_{z}=I_{0}\left|\left[j_{0}|1| j\right]\right|^{2} \frac{1}{\sqrt{2 j+1}} \rho_{0}^{0}(j) .
$$

In the same manner, for the difference between the intensities of radiation polarized in two mutually perpendicular directions (e.g., $O Z$ and $O Y$ ) and observed along the third direction (in this case $O X$ ), we have:

$$
\left(I_{z}-I_{y}\right)_{x}=I_{0}\left|\left[j_{0}|1| j\right]\right|^{2}(2 j+1)(-1)^{j+j_{0}+1}\left\{\begin{array}{ccc}
1 & 1 & 2 \\
j & j & j_{0}
\end{array}\right\}\left[\frac{3}{\sqrt{6}} \rho_{0}^{2}(j)+\operatorname{Re}\left(\rho_{2}^{2}(j)\right)\right] .
$$

For the difference between the intensities of radiation polarized along the right and left circle when observed along the axis $O Z$, we have:

$$
\left(I_{+}-I_{-}\right)_{z}=I_{0}\left|\left[j_{0}|1| j\right]\right|^{2}(2 j+1)(-1)^{j+j_{0}+1}\left\{\begin{array}{ccc}
1 & 1 & 1 \\
j & j & j_{0}
\end{array}\right\} \rho_{0}^{1}(j) .
$$

From the last three expressions, it can be seen, that the sum of the intensities of radiation polarized in three mutually perpendicular directions is defined by polarization moment of zero rank, the anisotropy of the radiation which is linearly polarized in two mutually perpendicular directions is determined by the components of the moment of the second rank, and, finally, the anisotropy of the circularly polarized radiation - by the components of the moment of the first rank. The polarization moment of the second rank is called alignment tensor, while the polarization moment of the first rank, which has three components - the orientation vector. Components $\rho_{0}^{2}$ of the alignment tensor is often named as longitudinal, whereas components $\rho_{1}^{2}$ and $\rho_{2}^{2}$ are known as uncleaned alignment and transverse ones. Based on the above, one can say that the polarization moments define the ordering of the angular momenta of the ensemble of particles and the anisotropy of the dipole radiation of that ensemble.

\section{Statement of the problem and discussion of the results of the numerical calculations}

In this paper, we consider the time dependance of $3{ }^{3} S_{1}$ state of the atom $\mathrm{He}$, the excitation of which is performed from $2^{3} P$ multiplet of this atom. It is assumed that the system is placed into a strong magnetic field sufficient to break the thin bond of P-multiplet under consideration. We will also assume that state $2^{3} P$ is populated from the ground state of this atom by a proton or electron impact or other axially symmetric excitation sufficient to tear up the fine bound, and which the symmetry axis makes an angle $\theta$ with the axis $O Z$ of the laboratory frame of reference. As a result of this excitation in the frame of reference associated with the anisotropy axis, on the $2^{3} P$ - levels, ordering of the angular moments of the population $T_{0}^{0}$ and longitudinal alignment $T_{0}^{2}$ will be induced. Then, by introducing a parameter $\alpha=T_{0}^{2} / T_{0}^{0}$, which may be called the anisotropy parameter, for efficiencies of populations of the magnetic sublevels of $2^{3} P_{j}$ state in the laboratory frame of reference $X Y Z$, one can write the following: 


$$
\sigma_{j, m ; j^{\prime}, m^{\prime}}=\frac{1}{2 j+1} A^{(0)}\left(j, j^{\prime}, l, s\right)+\alpha \sum_{q}(-1)^{j^{\prime}+m^{\prime}}\left[\begin{array}{ccc}
j & j^{\prime} & 2 \\
m & -m^{\prime} & q
\end{array}\right] D_{0, q}^{(2)}(\theta) A^{(2)}\left(j, j^{\prime}, l, s\right),
$$

where $l$ and $s$ are the orbital and the spin moments, $D_{m, m^{\prime}}^{2}(\theta)$ elements of three-dimensional rotation [13], and $A_{j}^{(k)}$ depends on coefficients of vector addition of moments:

$$
A^{(k)}\left(j, j^{\prime}, l, s\right)=(-1)^{j^{\prime}+s+k+l} \sqrt{(2 j+1)\left(2 j^{\prime}+1\right)}\left\{\begin{array}{lll}
l & l & k \\
j & j^{\prime} & s
\end{array}\right\} .
$$

The Hamiltonian operator of the atomic system placed in a strong magnetic field of strength $\mathcal{H}$ is defined by the orbital $\hat{l}$ angular momenta and $\hat{s}$ the spin ones

$$
\hat{H}=\hat{H}_{F}+\hat{W},
$$

where $\hat{H}_{F}=\hat{H}_{0}+\mu_{0} \hat{\mathcal{H}}(\hat{l}+2 \hat{s})$ depends on the value of the moments, $\mu_{0}$ - the Bohr magneton and on the value and direction of magnetic field. Furthermore, we expect that the direction of magnetic field makes an angle $\chi$ with the $O Z$ axis of the laboratory frame of reference.

Operator $\hat{W}$ describes the interaction of the system with the electromagnetic field, performing the excitation from $2^{3} P$ state to $3^{3} S$ ones (hereafter referred to as the state of ' $a$ ' and ' $b$ ', respectively (see Fig. 1)). Then, if we assume that the exciting field is linearly polarized along the axis $O Z$ of the laboratory frame of reference, in the basis of eigenfunctions of the square momentum and its projection for the matrix element of operator $\hat{W}$, it can be written as follows:

$$
\begin{gathered}
\left(W_{a b}\right)_{j_{a}, m_{a} ; j_{b}, m_{b}}=\left\langle j_{a} m_{a}\left|d_{z}\right| j_{b} m_{b}\right\rangle= \\
=\hbar \Omega(-1)^{l_{a}+s+j_{b}+l_{b}+1} \sqrt{\left(2 l_{b}+1\right)\left(2 j_{b}+1\right)}\left[\begin{array}{ccc}
j_{b} & 1 & j_{a} \\
m_{b} & 0 & m_{a}
\end{array}\right]\left\{\begin{array}{ccc}
l_{b} & l_{a} & 1 \\
j_{a} & j_{b} & s
\end{array}\right\}\left[\begin{array}{ccc}
l_{b} & 1 & l_{a} \\
0 & 0 & 0
\end{array}\right],
\end{gathered}
$$

where $\Omega=\mathcal{E}\left\langle j_{a}\|r\| j_{b}\right\rangle / \hbar$ is the Rabi frequency, which depends on the electric field intensity $\mathcal{E}$ and irreducible matrix element of operator $r$. The evolution of the density matrix for a system of two states is described by the Liouville equation, which for the blocks of the density matrix $\widetilde{\rho}_{i, j} i, j=a, b$ may be written in form:

$$
\begin{gathered}
\frac{d}{d t} \widetilde{\rho}_{a a}(t)=\Gamma \widetilde{\rho}_{b b}(t)-i\left(W_{a b}(t) \widetilde{\rho}_{b a}(t)-\widetilde{\rho}_{a b}(t) W_{b a}(t)\right), \\
\frac{d}{d t} \widetilde{\rho}_{b b}(t)=-\Gamma \widetilde{\rho}_{b b}(t)-i\left(W_{b a}(t) \widetilde{\rho}_{a b}(t)-\widetilde{\rho}_{b a}(t) W_{a b}(t)\right), \\
\frac{d}{d t} \widetilde{\rho}_{a b}(t)=-\frac{\Gamma}{2} \widetilde{\rho}_{a b}(t)-\frac{i}{\hbar}\left(E_{a}-E_{b}\right) \widetilde{\rho}_{a b}(t)-i\left(W_{a b}(t) \widetilde{\rho}_{b b}(t)-\widetilde{\rho}_{a a}(t) W_{a b}(t),\right. \\
\widetilde{\rho}_{b a}(t)=\widetilde{\rho}_{a b}^{\dagger}(t),
\end{gathered}
$$

where $\Gamma$ is a matrix that describes relaxation processes. The solution of this system should be found according to initial conditions $\widetilde{\rho}_{b b}(0)=0, \widetilde{\rho}_{a b}(0)=0, \widetilde{\rho}_{b a}(0)=0$. As to density matrix of the state ' $a$ ', in the base of the eigenfunctions of the square of the momentum and its projection this matrix is proportional to the matrix $\sigma_{j, m ; j^{\prime}, m^{\prime}}$, which is specified by relation (9). In order to reduce the system (13) to a more convenient form for numerical computation, first, it is necessary to introduce dimensionless time $\tau=t / \tau_{0}$, where $\tau_{0}-$ the 
lifetime of the excited $3^{3} S_{1}$ state, and secondly, to move from the basis of eigenfunctions of the square of the momentum and its projection to the basis on which operator $H_{F}$ of (11) is diagonal ( $M$-basis). Then, if we introduce density matrices $\rho_{i, j}(t)(i, j=a, b)$, connected with the similar matrices introduced earlier with the help of relations $\left(\rho_{i j}(\tau)\right)_{M, M_{1}}=\left(\widetilde{\rho}_{i i}(\tau)\right)_{M, M_{1}}$ $(i=a, b),\left(\rho_{b a}(\tau)\right)_{M, M_{1}}=\left(\widetilde{\rho}_{b a}(\tau)\right)_{M, M_{1}} \exp \left[-i \tau\left(\left(E_{b}\right)_{M}-\left(E_{a}\right)_{M_{1}} / \hbar\right)\right]\left(\rho_{i, j}(\tau)=\left(\rho_{j, i}(\tau)\right)^{\dagger}\right)$, then the dimensionless energy levels $\left(E_{a}\right)_{M}=E_{a}+\left(\varepsilon_{a}\right)_{M}$ and $\left(E_{b}\right)_{M}=E_{b}+\hbar \omega+\left(\varepsilon_{b}\right)_{M}$ are expressed through $\left(\varepsilon_{i}\right)_{M}(i=a, b)$ - the eigenvalues of operator $\hat{H}_{F}$ and $\omega=\left(E_{b}-E_{a}\right) / \hbar-$ transition frequency between the levels of $b$ and $a$ with the energies $E_{b}$ and $E_{a}$ respectively (see Fig. 1) and, at last the laser frequency $\omega_{e x}=\omega+\widetilde{\delta} / \hbar$. The eigenvalues $\left(\varepsilon_{a}\right)_{M}$ and $\left(\varepsilon_{b}\right)_{M}$ here possess the values $\pm n \mu_{0} \mathcal{H}$, whereas $(n=0,1,2,3)$ and $(n=0,2)$ for the states ' $a$ ' and ' $b$ ' respectively. It should be mentioned that eigenvalue $n=1$ of the state ' $a$ ' is twice degenerate.

Using these new notations, the system (13) takes the form:

$$
\begin{gathered}
\frac{d}{d \tau} \rho_{a a}(\tau)=\gamma_{a} \rho_{b b}(\tau)+\frac{i}{2 \hbar} e^{-i \tau \delta} \rho_{a b}(\tau)\left(W_{b a}\right)_{I}(\tau)-\frac{i}{2 \hbar} e^{i \tau \delta}\left(W_{a b}(\tau)\right)_{I} \rho_{b a}(\tau), \\
\frac{d}{d \tau} \rho_{b b}(\tau)=-\gamma \rho_{b b}(\tau)-\frac{i}{2 \hbar} e^{-i \tau \delta}\left(W_{b a}\right)_{I} \rho_{a b}(\tau)+\frac{i}{2 \hbar} e^{i \tau \delta} \rho_{b a}(\tau)\left(W_{a b}\right)_{I} \\
\frac{d}{d \tau} \rho_{a b}(\tau)=-\frac{\gamma}{2} \rho_{a b}(\tau)+\frac{i}{2 \hbar} e^{i \tau \delta} \rho_{a a}(\tau)\left(W_{a b}\right)_{I}-\frac{i}{2 \hbar} e^{i \tau \delta_{a}}\left(W_{a b}\right)_{I} \rho_{b b}(\tau) \\
\frac{d}{d \tau} \rho_{b a}(\tau)=-\frac{\gamma}{2} \rho_{b a}(\tau)-\frac{i}{2 \hbar} e^{-i \tau \delta}\left(W_{b a}\right)_{I} \rho_{a a}(\tau)+\frac{i}{2 \hbar} e^{-i \tau \delta_{a}} \rho_{b b}(\tau)\left(W_{b a}\right)_{I}
\end{gathered}
$$

where the subscript ' $I$ ' indicates that this operator is recorded in the interaction representation $\left((W a b)_{I}\right)_{M, N}=\left(W_{a b}\right)_{M, N} \exp \left[i\left(\left(\varepsilon_{a}\right)_{M}-\left(\varepsilon_{b}\right)_{N}\right) \tau\right]$ and $\delta$ is the dimensionless laser detuning $\delta=\widetilde{\delta} \tau_{0} / \hbar$

It should be noted that the $2^{3} P$ state, owing to the transition from the level with $j=1$ (when level with $j=2$ is metastable one [15]), is characterized by the finite value of the lifetime. This leads an exponential decrease in the value $\sigma_{j, m ; j^{\prime}, m^{\prime}}(9)$ and for additional exponential decreasing of the solution of the system (13), that did no connect with the coherence process. Therefore, one can say that the decrease in the population of the $2^{3} P$ state is connected with the presence of magnetic field, that mixes the levels of this multiplet. This exponential decay will not be considered in this article.

With regard to the integration of the system of matrix equations, the left side of which contains a matrix of order $n$, it should be noted, that the universal method in this case is a method of reducing this system to the system of ordinary differential equations of order $n^{2}$. In the case when the matrix coefficients of the right part do not depend on the time, the system can be integrated using the Laplace transform or the transition from the matrix $\widetilde{\rho_{i, j}} i, j=a, b$ , to the matrix $\rho=U^{\dagger} \widetilde{\rho} U$ [16], where the matrix $U$ - is the matrix of the eigenvectors of the Hamiltonian operator (11). In the present paper, system (13) was integrated by the series expansion, the implementation of which may be briefly described as following. Due to the fact that the density matrix does not depend explicitly on the time, the derivative of the matrix $\widetilde{\rho}_{i, j}$ of order $n$, can be obtained from the derivative of order $n-1$ of this matrix by using the commutation of the latter matrix with the Hamiltonian operator (11). After this, for the derivative of $\widetilde{\rho}_{i, j}^{(n)}$, expressions similar to (13), with the difference that the right side contains derivatives of $\widetilde{\rho}_{i, j}^{(n-1)}$ will be obtained. After this, performing replacements similar to those mentioned above, leads to expressions for the derivatives of the matrix $\rho_{i, j} i, j=a, b$, that are similar to (14). Using such procedure, at a fixed time, one can calculate the first four derivatives 


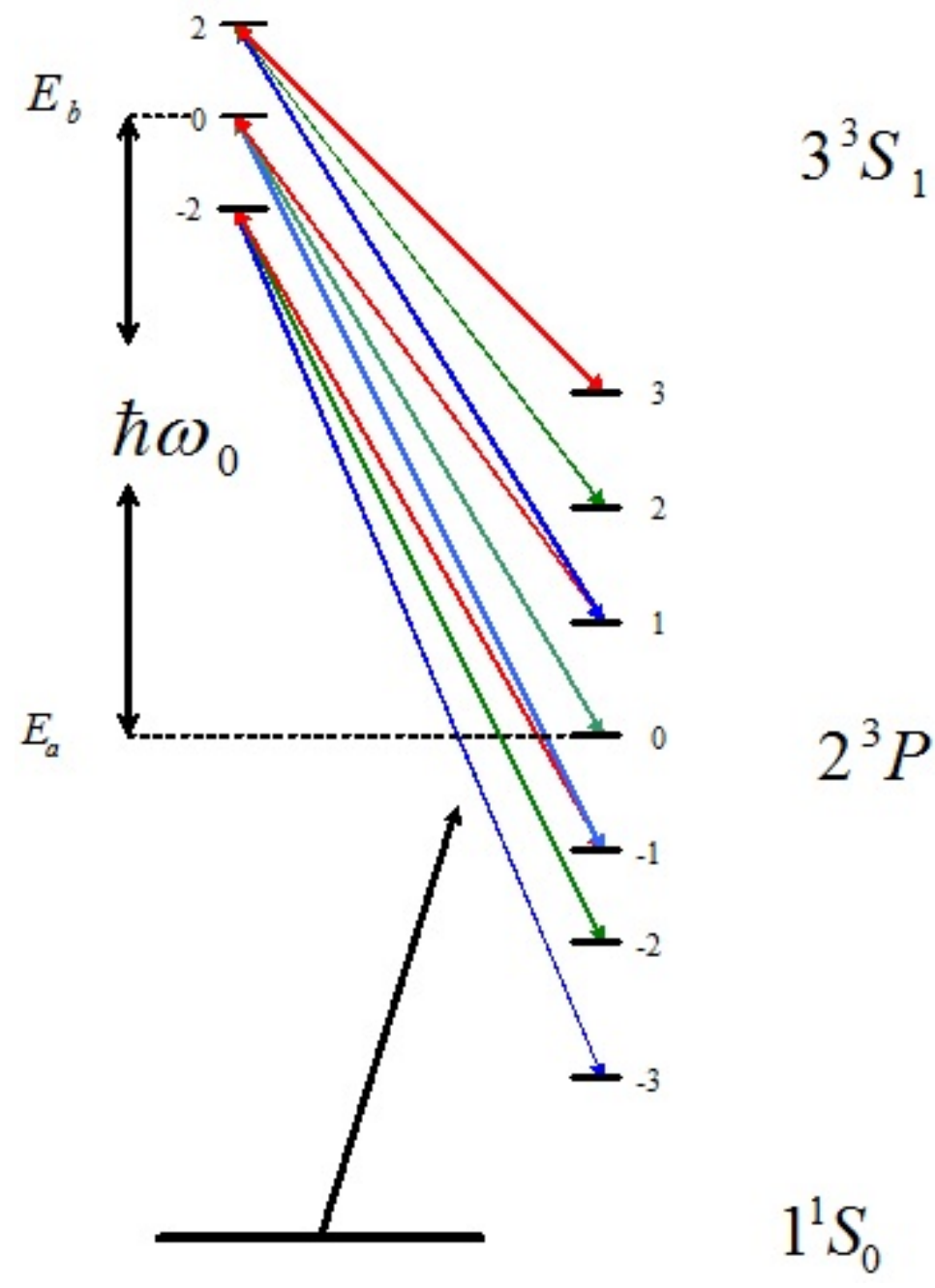

FIG. 1. Splitting scheme of $3^{3} S$ and $2^{3} P$ states of the He atom in a strong magnetic field ( $M-$ base). (Eigenvalues \pm 1 of state ' $a$ ' are doubly-degenerate).

of the matrix $\rho_{i, j}$, and then determine the value of this matrix at the incremented point using the Taylor formula. As for the relaxation terms, they were considered in accordance with the Pauli equation [15]: as the level $3^{3} S_{1}$ decays incrementally into multiplet levels of $2^{3} P$, a term describing the relaxation of state ' $b$ ' has the form of $\frac{1}{3} I_{3} \rho_{b b}$, where $I_{3}$ is the identity matrix of the third order. (The time is measured in units of the time of life of $3^{3} S_{1}$ state and therefore the time interval equal to the lifetime of this state is equal to one). As for the density matrix of the multiplet $2^{3} P$ (state ' $a$ '), because of the proposed isolation of the $3^{3} S_{1}$ and $2^{3} P$ states, the term in equation (14) describing the relaxation of the state ' $a$ ' has the form $\frac{1}{9} I_{9} \rho_{a a}$, where $I_{9}$ is the identity matrix of the ninth order. With regards to the correlation density matrices, in this paper it is assumed that their relaxation constants are $\gamma / 2$.

At Fig. 2 the dependency of the population of $3^{3} S_{1}$ state of $\mathrm{He}$ atom on the dimensionless time for several values of the detuning of the laser frequency $\delta=0,1,2,3$, the intensity of magnetic field $H=5000 G$ and the angle of inclination of magnetic field direction $\chi=\pi / 4$ is represented. From this picture, it can be inferred that the CPT state is achieved for sufficiently large frequency deviations $(\delta \geq 2)$. The latter fact is clear from the above, since the entire chain of $\Lambda$ - schemes 'opens' only for sufficiently high $\delta$. 


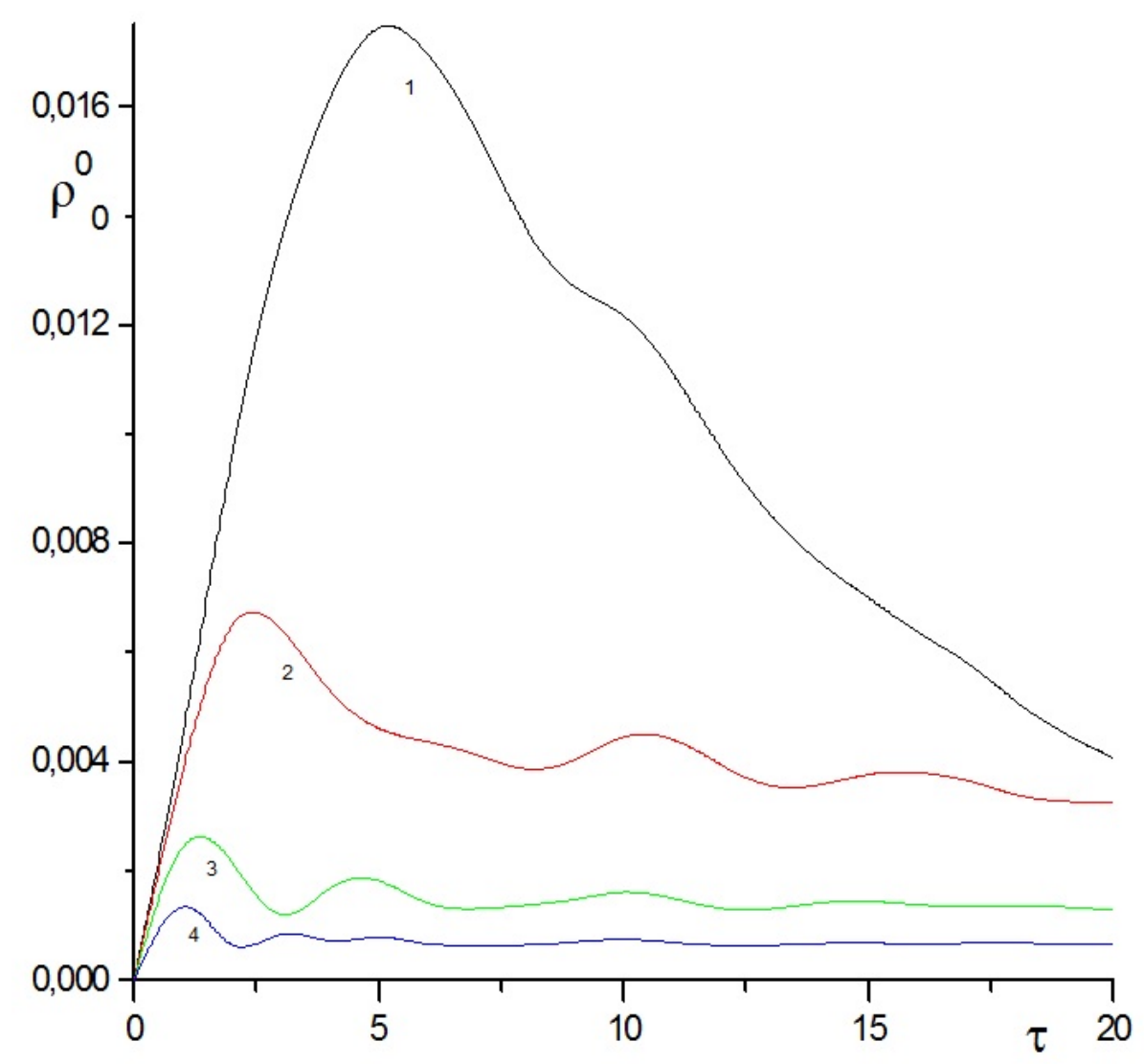

FIG. 2. The dependence of the $3^{3} S$ state population of the He atom on dimensionless time at $\mathcal{H}=5000 G$ and $\chi=\pi / 4$ for $\delta=0,1,2,3$ (lines $1-4$ respectively)

We note further, that under the axially symmetric impact excitation of $2^{3} P$ multiplet, at the ${ }^{3} S_{1}$ level, alignment is not at all induced for all values of the angles $\chi$ and $\theta$. This result seems complex: the $3^{3} S_{1}$ state is populated from levels of the $2^{3} P$ multiplet, where the impact alignment is induced, and therefore, the anisotropy of the lower state population should be transferred to the upper states.

Such a situation is shown in Fig. 3, where the dependence of the alignment induced in the $3^{3} S_{1}$ state while separate isotropic population of the $2^{3} P_{1}$ and $2^{3} P_{2}$ levels when $\delta=2$ is illustrated. The last figure shows that alignment, which is induced on the ${ }^{3} S_{1}$ state, is of different sign when population of the $2^{3} P_{2}$ or $2^{3} P_{1}$ state is occurring. Dependence of the alignment induced from the ${ }^{3} P_{2}$ state on time changes sign from negative to positive, however, the case is reversed for the ${ }^{3} P_{1}$ state. It is interesting that for each population mean, the sign will change at the very same instant. From the above, it can be concluded that the signal of alignment becoming zero is due to both the symmetry of the impact-excited $2^{3} P$ state and that of the $W_{a b}$ matrix, which describes the excitation process of the upper $3^{3} S_{1}$ level. It should be mentioned, that the above-represented reasoning only illustrates the inputs of states with $j=1$ and $j=2$ of multiplet $2^{3} P$ into alignment induced on the $3^{3} S_{1}$ state: in the presence of a strong magnetic field, states with different $j$ are mixed and separate excitation from these states is impossible.

Figure 4, shows the dependency of the $3^{3} S_{1}$ state population on dimensionless time at a fixed detuning, intensity of the magnetic field and for the several values of angle $\chi$. The figure 


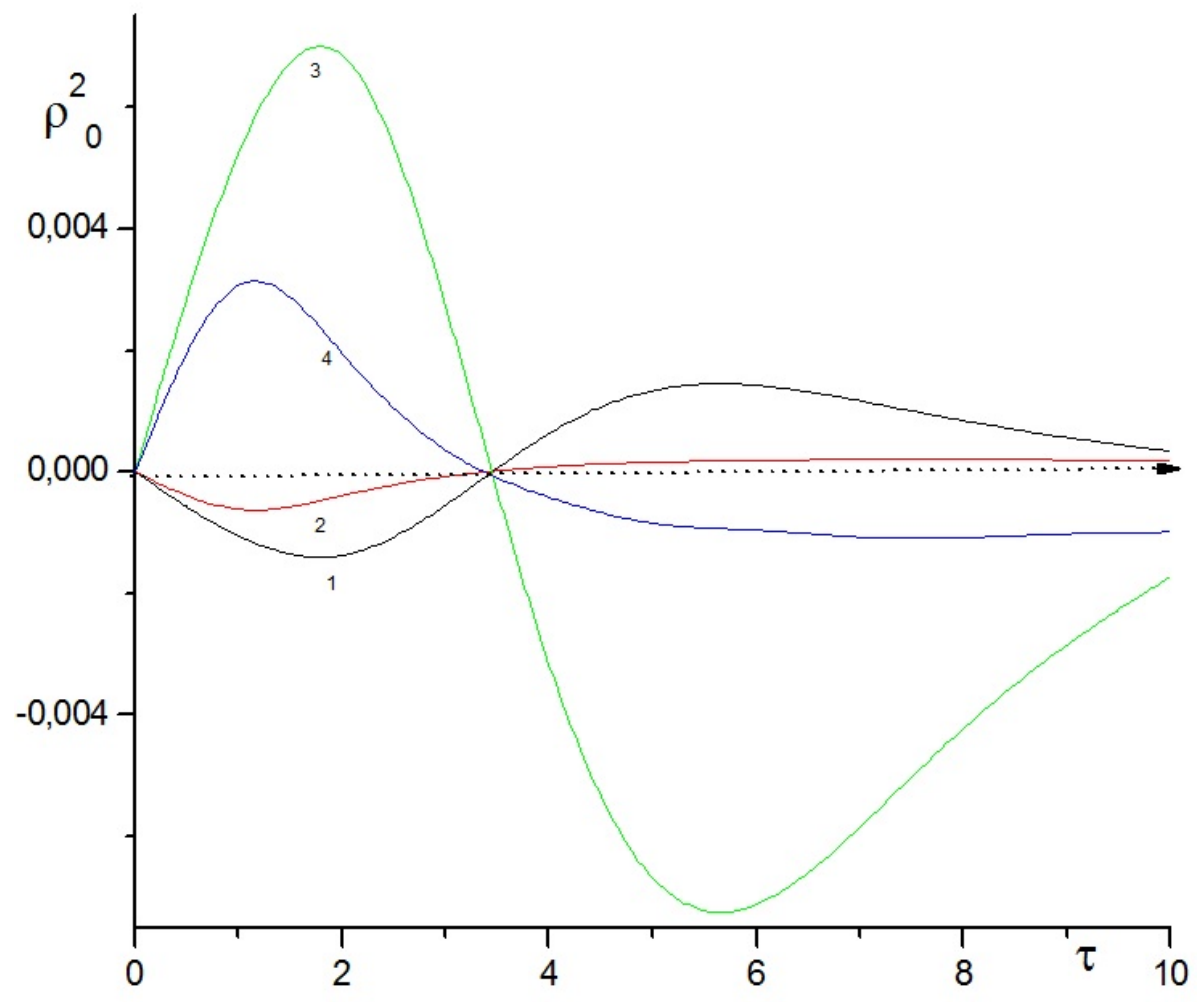

FIG. 3. The dependence of the alignment induced on $3^{3} S_{1}$ state, when population happens individually for the $2^{3} P_{2}$ (curves 1 and 2) and $2^{3} P_{1}$ (curves 3 and 4) levels $(\delta=1,2, \mathcal{H}=5000 G$ and $\chi=\pi / 4)$.

shows that the population increases with the angle $\chi$ increasing from 0 to $\pi / 4$, but then begins to decrease. This decreasing becomes faster the closer the angle $\chi$ is to the value of $\pi / 2$. When $\chi=\pi / 2$, the population turns to zero. Note that a similar result was obtained in [17] in the framework of approximate solutions for the Schrodinger equation with the use of perturbation theory over the Rabi frequency. Using a similar method for the approximate solution of system (14) has led to the following expression for the matrix elements $\rho_{b b}$ :

$$
\begin{gathered}
\left(\rho_{b b}\right)_{M, M_{1}}=\hbar^{2} \sum_{\xi, \mu}\left(W_{b a}\right)_{M, \xi}\left(\rho_{a a}(0)\right)_{\xi, \mu}\left(W_{a b}\right)_{\mu, M_{1}} \\
{\left[\exp \left(\frac{\left(-\gamma \hbar-2 i\left(\delta+\left(\varepsilon_{a}\right)_{\xi}-\left(\varepsilon_{b}\right)_{M}\right)\right.}{2 \hbar} t\right)+\exp \left(\frac{\left(-\gamma \hbar+2 i\left(\delta+\left(\varepsilon_{a}\right)_{\mu}-\left(\varepsilon_{b}\right)_{M_{1}}\right)\right.}{2 \hbar} t\right)-\right.} \\
\left.\exp \left(\frac{\left(-\gamma \hbar+2 i\left(+\left(\varepsilon_{a}\right)_{\mu}-\left(\varepsilon_{a}\right)_{\xi}+\left(\varepsilon_{b}\right)_{M}-\left(\varepsilon_{b}\right)_{M_{1}}\right)\right.}{2 \hbar} t\right)\right]
\end{gathered}
$$

which, due to cumbersome analytical expressions for $W_{a b}$ and $\rho_{a a}(0)$ in $M$-basis, is rather short at only $\chi=\pi / 2$, when for the population of level $b$ one can write the following: 


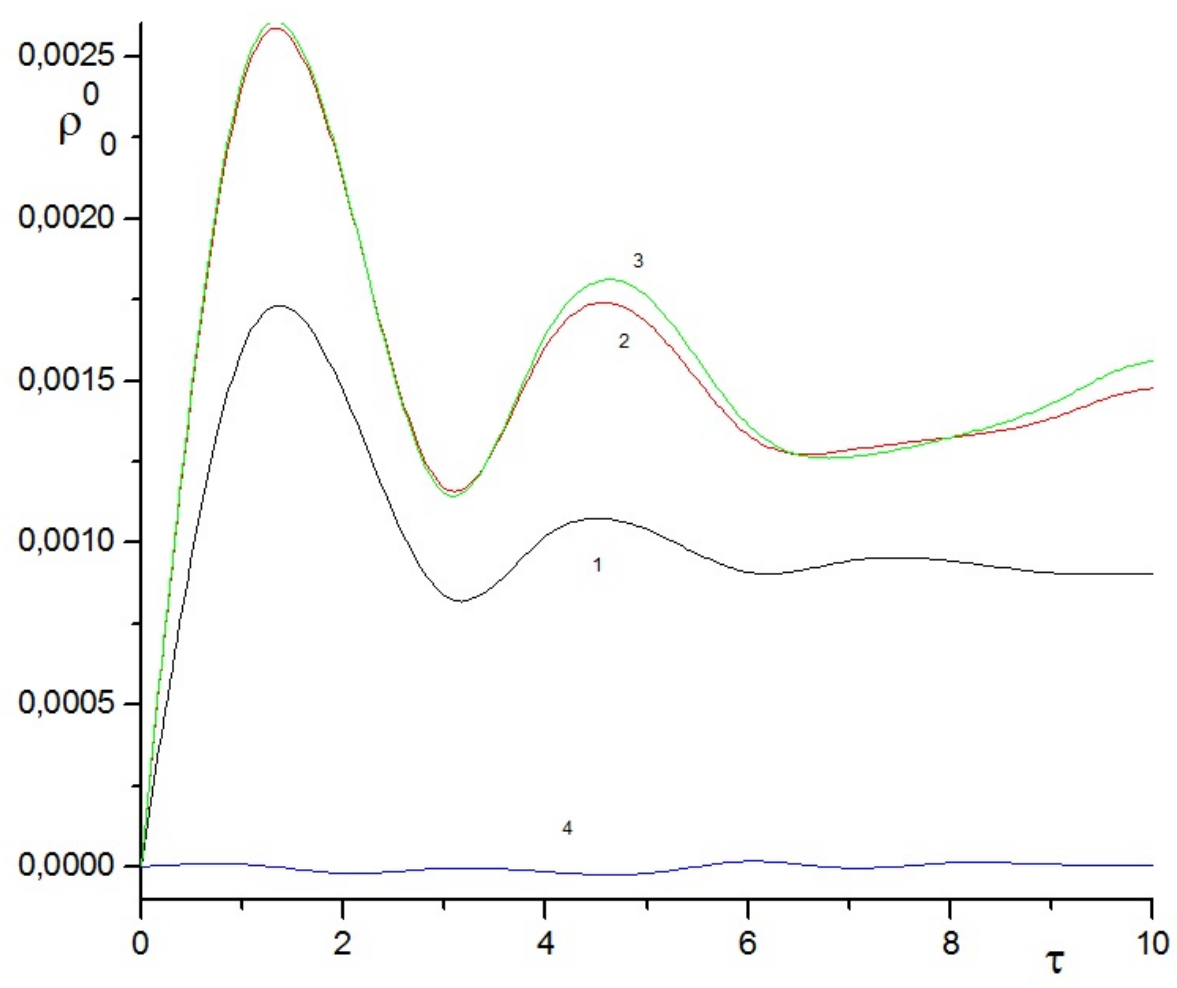

FIG. 4. The dependence of the population of $3^{3} S$ He atom on the dimensionless time at $\mathcal{H}=5000 G, \delta=2$ and $\chi=0, \pi / 6, \pi / 4, \pi / 2$ (lines $1-4$ respectively).

$$
\begin{gathered}
\left(\rho_{b b}\right)_{0}^{0}(t)=\frac{\hbar^{2}}{288 \sqrt{3}\left(\Omega_{L}^{2}-\delta^{2}\right)^{2}}\left[-11 \delta^{2}-17 \Omega_{L}^{2}+4\left(5 \Omega_{L}^{2}+2 \delta^{2}\right) \cos \left(\frac{\delta t}{\hbar}\right) \cos \left(\frac{\Omega_{L} t}{\hbar}\right)+\right. \\
\left.3\left(\delta^{2}-\Omega_{L}^{2}\right) \cos \left(\frac{2 \Omega_{L} t}{\hbar}\right)+28 \delta \Omega_{L} \sin \left(\frac{\delta t}{\hbar}\right) \sin \left(\frac{\Omega_{L} t}{\hbar}\right)\right]
\end{gathered}
$$

From the last expression, where, for brevity, we set $\gamma=0$, one can see that for large values of the magnetic field intensity $\left(\rho_{b b}\right)_{0}^{0} \sim 1 / \Omega_{L}^{2}$, which is consistent with the results of numerical calculation, which, let us recall, provides the populations turning to zero at $\chi=\pi / 2$. Regarding the alignment, the approximate calculation according to equation (16) shows that at the point $\chi=\pi / 2$, it has the highest infinitesimal order than $1 / \Omega_{L}^{2}$, which is also consistent with the results of numerical calculations.

We further note that when $\chi \neq 0$, the magnetic field reduces the symmetry of the problem, resulting in the possibility of induction at the $3^{3} S$ level, the ordering of types different from the populations and longitudinal alignment discussed above. Numerical calculations showed that the character of the of the uncleaned and transverse alignment dependencies, $\left(\rho_{b b}\right)_{1}^{2}$ and $\left(\rho_{b b}\right)_{2}^{2}$, respectively are the same: they first increase and then decrease while remaining positive over the $0<\chi<\pi / 2$ interval, and are equal to zero at the ends of this interval. An example of an uncleaned alignment depending on the time for several values of the detuning of the laser frequency is shown in Fig. 5. As for orientation, it also becomes zero at the ends of said interval, whereas on the inside it is about two orders of magnitude smaller than the alignment. 


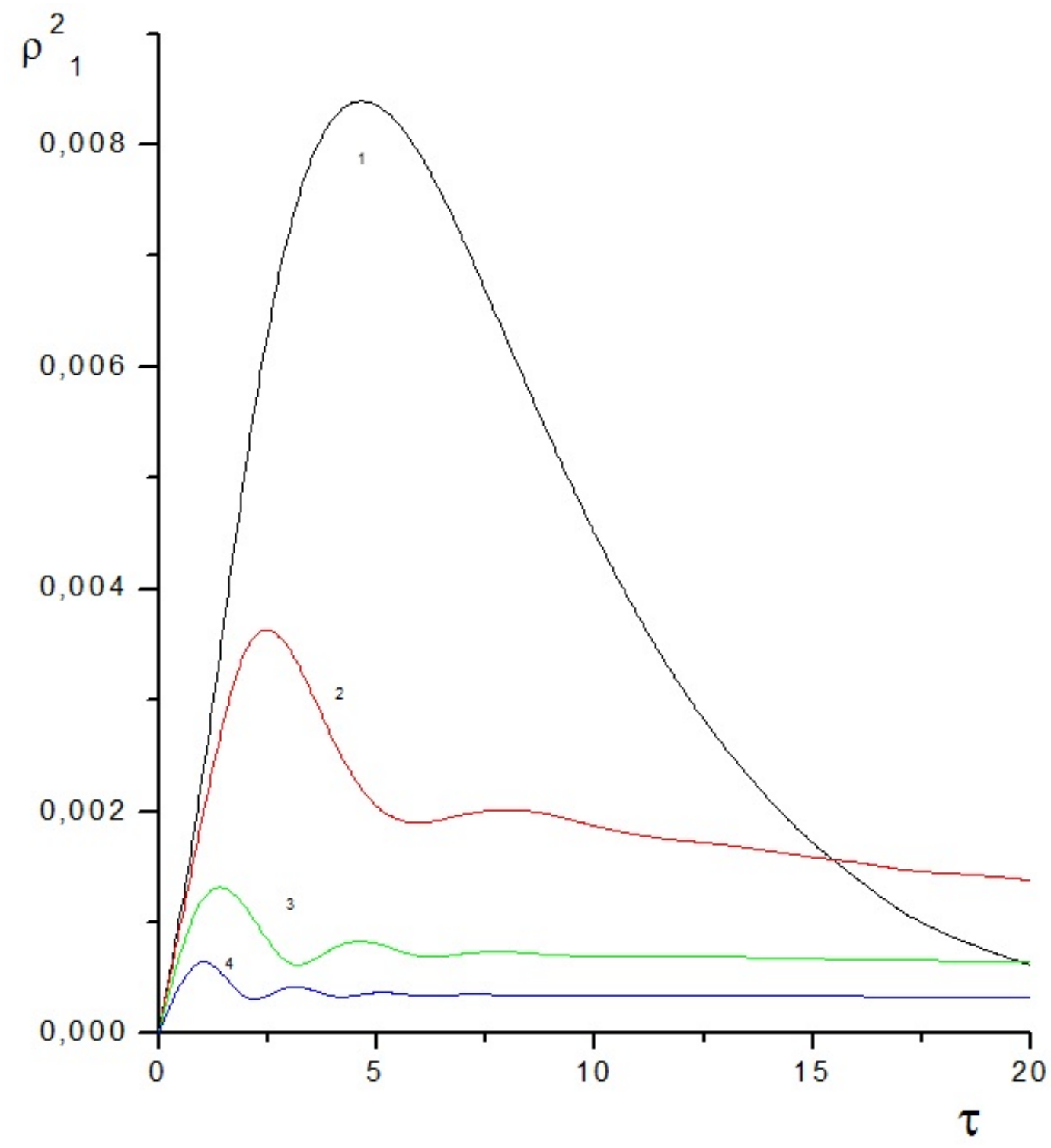

FIG. 5. The dependence on the uncleaned alignment of dimensionless time at $\mathcal{H}=5000 G, \chi=\pi / 4$ and $\delta=0,1,2,3$ (lines $1-4$ respectively)

\section{Conclusion}

In this paper, the helium atom is used as an example for investigation of the influence of a strong magnetic field on the polarization characteristics of the radiation of a multi-level system, a set of lower levels of which are populated from the ground state by an axially symmetric excitation, while the upper levels are populated from lower levels in a coherent manner. With the help of numerical solutions of the Liouville equation, it has been shown that the system achieves the CPT state for large enough detunings of the laser frequency. For lower detunings at the excited state of the system, angular momenta ordering of population type is induced, whereas the ordering of alignment type is absent. In cases when the direction of the excitation processes anisotropies are orthogonal to the magnetic field direction, the system reaches a state of CPT, i.e. population, and hence the alignment of the system turns to zero. 


\section{References}

[1] M. Scully, S. Zhu, A. Cavrielides. Degenerate Quantum-Beat Laser: Lasering without inverion and invertion without lasering. Phys. Rev. Lett., 62, P. 2813-2816 (1982).

[2] F. Cruz, M.L. Sundheimer, W.C. Magno. Temperature limits in laser cooling of the atoms with three-level cascade transitions. Phys. Rev. A, 87, P. 063409,(2013).

[3] D. Moretti, D. Felinto, J.W.R. Tabosa, A. Lezama. Dynamics of the stored Zeeman coherence grating in the external magnetic field. J. Phys. B, 43,P. 11550 (2010).

[4] S.A. Kazantsev, A.G. Petrashen, V.N. Rebane, T.K. Rebane. Anisotropic collisional relaxation of polarization moments and self-alignment of ions in a plazma. Zh. Eksp. Teor. Fiz., 106, P. 698-724 (1994).

[5] S.A. Kazantsev, A.G. Petrashen, N.M. Firstovs, J.-C. Henoux. The determination of the energy of electron beam with help of spectropolarimetric inverstigation of emission of Ellerman's bombs. Opt. i Spektr., 86 (4), P. 559-562 (1999).

[6] S.A. Kazantsev, A.V. Michalev, A.G. Petrashen. The spectroscopy of the upper levels of atmosphere. Polarization of the optical flares in the emission of the night sky. Opt. i Spektr., 82 (4), P. 714-722 (1996).

[7] A.S. Shlyaptseva, S.B. Hansen, et al. The spectropolarimetry of high temperature plazma. Rev. San. Instrum., 72 (1), P. 1241-1244 (2001).

[8] S.A. Kazantsev, A.G. Petrashen. The diagnostics of the magnetic field of tokomac's high temperature plazma with methods of polarization spectoscopy. Opt. i Spektr, 90 (1), P. 30-32 (2001).

[9] S.A. Kazantsev, A.G. Petrashen, M.I. Pudpovkin. The diagnostics of local magthtic fields by methods of polarization spectroscopy. Aeron. i Geomagnet., 41 (1), P. 50-56 (2001).

[10] S.A. Kazantsev, A.G. Petrashen, N.M. Firstova. Impact Spectropolarimetric Sensing. Physics of atoms and molecules. Kiuwer Academic/Plenum Publishes, 1999.

[11] P. Valente, Y. Failache, A. Lezama. Comparative of transient evolution of Hanle inducend transparency and absorbtion resonance. Phys. Rev A, 65, P. 023814 (2002).

[12] M.U. Momeen, O. Rasgarajan, V. Nastorajan. Transient response of nonlinear magneto-ohtics radiation in a paraphin-coated $R b$ vapor sell. Phys. Rev A, 81, P. 013453 (2010).

[13] E. ElBaz, B. Cactel. Grarhical methods of spin Algebras, Marcel Dekker Inc., New York, 1972.

[14] A. Radzig, B.M. Smirnov. Reference data on atoms, molecules and ions, Springer-Verlag, Berlin, 1985.

[15] K. Blum. Density Matrix Theory and Applications, Plemnum Press New York and London, 1981.

[16] Kai-Shue-Lam. Coherent laser exitation of ${ }^{137} B a$ and ${ }^{138}$ Ba. Phys. Rev. A, 45 (5), P. 3084-3092 (1992).

[17] A.G. Petrashen, N.V. Sytenko. Polarization phenomna under the coherent excitation at the pesence strong madnetic field. Nanosystems: Phys. Chem. Math., 3 (5), P. $62-69$ (2012). 